\title{
Partial vertebrectomy with vertebral shortening for thoraco-lumbar fracture-dislocation. Case report and technical note
}

\author{
Bogdan Costachescu, ${ }^{1,2}$, Cezar E. Popescu' ${ }^{1}$, Bogdan Iliescu ${ }^{1}$, \\ Alexandru Chiriac ${ }^{1}$, Ramona Filipescu ${ }^{3}$, Liviu Pendefunda ${ }^{4}$ \\ ${ }^{1}$ Department of Neurosurgery, Emergency University Hospital “Prof. Dr. N. Oblu”, Iasi \\ ${ }^{2} \mathrm{PhD}$ student University of Medicine and Pharmacy "Grigore T. Popa" Iasi \\ ${ }^{3}$ Department of Neurosurgery, Emergency Pediatric University Hospital “Sfanta Maria”, Iasi \\ ${ }^{4}$ Department of Neurology, Emergency University Hospital “Prof. Dr. N. Oblu”, Iasi
}

\begin{abstract}
Thoraco-lumbar fracture-dislocations represent one of the most instable lesions and are frequently associated with neurological deficit. We present a patient with a T11 - T12 fracture-dislocation with complete neurological deficit - ASIA - A, who underwent partial vertebrectomy, shortening of the spine and posterior instrumentation 21 days after a motor vehicle accident.

Key words: thoraco-lumbar fracturedislocation, spine fracture, partial vertebrectomy, spine shortening.

\section{Introduction}

Thoraco-lumbar fracture-dislocations represent one of the most instable lesions and are frequently associated with neurological deficit (1). This type of lesion is not very common representing only $16 \%$ of thoracolumbar fractures. (2), but are very often associated with other lesions which could delay the timing of spine surgery. The treatment of choice for this lesion, whether
\end{abstract}

anterior, posterior, or combined approaches is still controversial. (3).

In this paper we report a case of T11 - T12 fracture-dislocation with complete neurological deficit, and we discuss the surgical treatment, presenting some technical notes.

\section{History and examination}

A 16-years-old boy presented in our Emergency Department with complete paraplegia and chest pain few hours after a motor vehicle accident (passenger without seat belt). Neurological exam showed complete neurological deficit (paraplegia, no voluntary anal contraction, no anal sensation S4, S5 sensory scores ) ASIA-A. Spine exam showed thoraco-lumbar segmental kyphosis with swollen soft tissues.

Imaging - whole body CT scan depicts T11-T12 fracture-dislocation with fractures of the posterior arch of T11, and T12 (fig. 1, 2), bilateral hemothorax and right pulmonary contusion. 


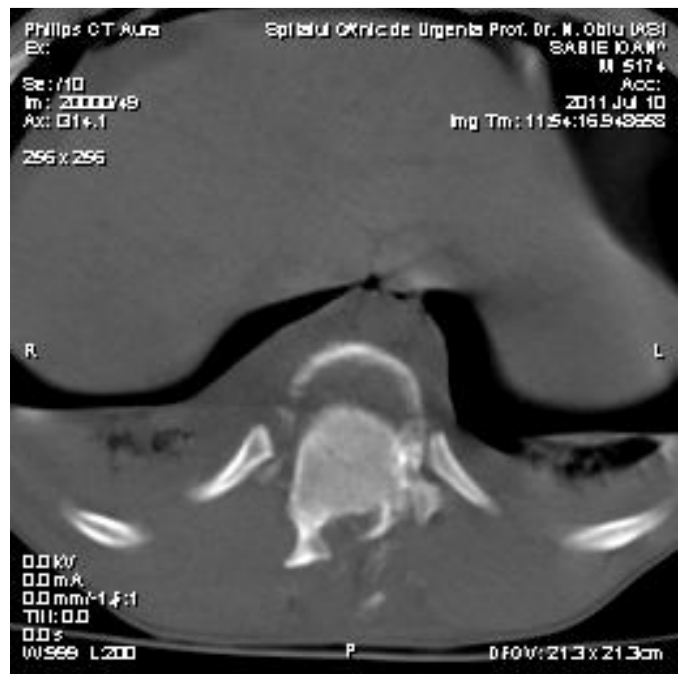

Figure 1 - axial image - T11-T12 dislocation with fractures of posterior arch T11 and T12

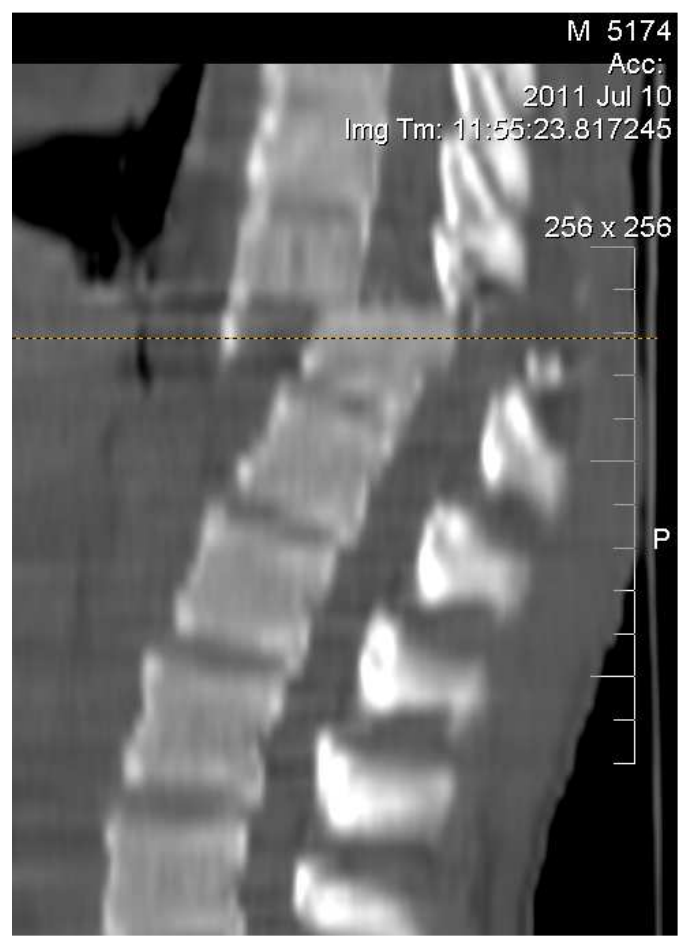

Figure 2 - sagittal reconstruction - T11-T12 dislocation

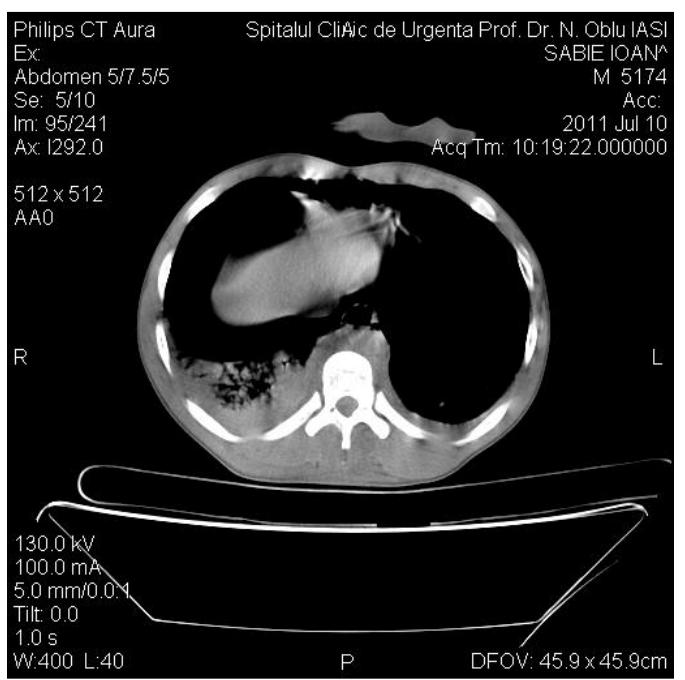

Figure 3 - axial image of thorax with bilateral hemothorax and left pulmonary contusion

The patient was admitted in the Department of Pediatric Surgery for his respiratory issues. He was subjected to spine surgery 21 days after the accident when the neurological status was unchanged - ASIA-A.

Surgical technique

We performed partial vertebrectomy with spine shortening in the manner described by Reyes -Sanchez in 2002 for thoraco-lumbar fractures (4).

Under general anesthesia the patient was placed in prone position. Through a midline incision the muscles are detached in subperiosteal fashion, exposing the laminae from T9 to L2. The dissection was carried out laterally to the tip of the transverse processes; the anterior dislocation was obvious. First there were removed the spinous processes and the laminae T11 and T12. There was a posterior dural tear of $4 \mathrm{~mm}$ thorough which instead of CSF, there was a "leakage" of spinal cord lacerated tissue. Under fluoroscopic 
guidance the screws are inserted bilaterally in the pedicles of T9, T10, L1 and L2.

Under magnification (loupes $\mathrm{x} 3$ ) the facet joints T11-T12 were removed. Using a highspeed drill the upper part of the T12 pedicles were resected giving access to the superior two thirds of the vertebral body which is emptied. Using rongeurs and forceps T11 - T12 disc is taken out. The inferior plateau of T11 is prepared for arthrodesis. The reduction was achieved bending the two rods to restore the anatomy of the sine in sagittal plane and attached first to inferior screws; using the persuader the rods were attached at the end to the superior screws. The screws are tightened in compression in order to build up a firm contact between inferior aspect of T11 vertebral body and the remaining one third of T12 body. Cancellous bone was impacted at this level for fusion. Finally the dural tear is sutured with 6-0 Prolene and packed with Gelfoam. The duration of surgery was 190 minutes.

Postoperative outcome was uneventful. The neurological findings remain unchanged. Plain X-rays depict partial reduction of the dislocation and the maintaining of almost normal curvatures of thoraco-lumbar region.

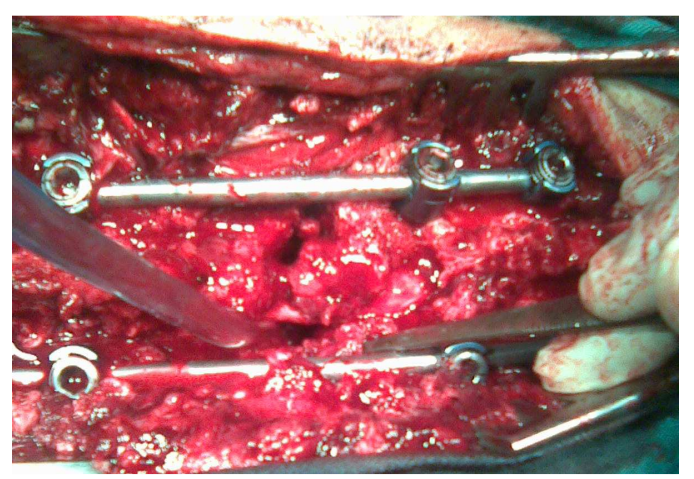

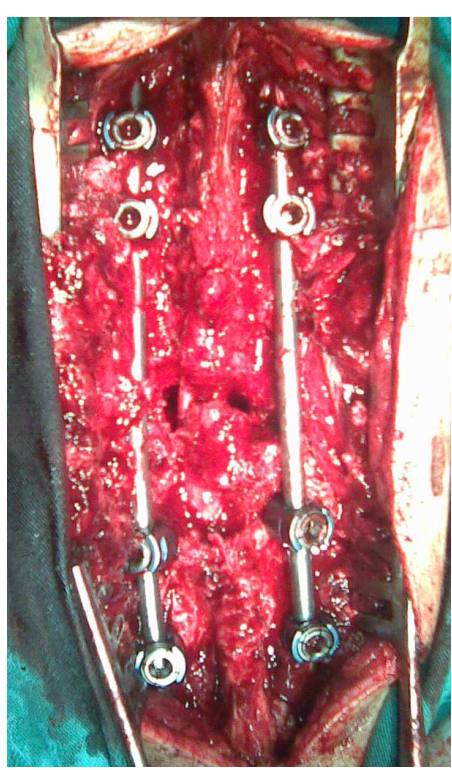

Figures 4 and 5 - intraoperative images; the suction (inferior left) is spotting the vertebrectomy. Note the absence of buckled dura.

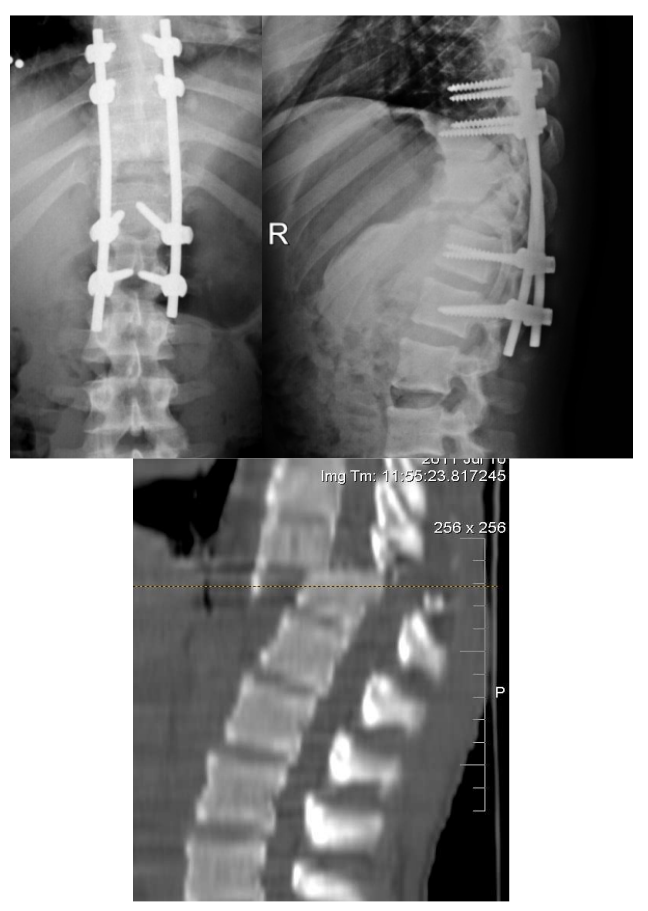


Figure 6 - plain X-rays AP (left), profile (middle) and comparison with preoperative image (right).

\section{Discussion}

Thoraco-lumbar fracture-dislocations are one of the most instable traumatic lesions disrupting all of three columns (5), frequently associated with neurological deficit. In patients with complete neurological deficit the goal of surgery is to reduce the dislocation and to provide a stable spine allowing early rehabilitation. A lesion as we described with dural tear and lacerated spinal cord tissue has less chances to improve even if we achieved partial reduction and performed decompression.

The approach for this lesions with disruption of posterior ligament complex due to anterior dislocation associated with complete neurological deficit should be posterior (6). Some authors advocate the anterior approach for these lesions but there are many complications worsened by the other posttraumatic lesions such as bilateral hemothorax and left pulmonary contusion in our case (7).

Partial vertebrectomy with vertebral shortening for thoraco-lumbar burst fracture was described by Reyes-Sanchez in 2002 (4) in order to treat by posterior approach the lesions which need anterior one. We also used this method for our case because there were 21 days after the accident and we presumed that the reduction without partial vertebrectomy will fail. The surgery for fracture- dislocation must be done in less than three weeks in order to obtain an acceptable reduction. (8)
In the same time we could consider performing the same shortening by using total vertebrectomy and fusion as described in 2011 by Obeid (9). There were some reasons for not using these technique:

- Our case presented only partial dislocation and not complete one as the cases presented in this paper, so we assumed that we can achieve appropriate reduction

- We could avoid dural buckling removing only two thirds of the vertebral body. In case of complete vertebrectomy we should cut the dural sac and close it separately

- Our instrumentation was shorter - 8 screws compared with minimum 12 screws used in total vertebrectomy decreasing the time of surgery, blood loss and the number of vertebral levels blocked.

- Total vertebrectomy is a surgery with important complications rate (10).

\section{Conclusions}

Partial vertebrectomy with vertebral shortening is a reliable technique for thoracolumbar fracture-dislocation with complete neurological deficit allowing in one step good reduction and fusion for early rehabilitation It should be performed as soon as possible after the injury (before three weeks) for a good reduction of dislocation.

\section{References}

1. Vaccaro AR, Kim DH, Brodke DS et al. Diagnosis and management of thoraco-lumbar spine fractures. Instr. Course Lect. 2004;53: 359-373.

2. Gertzbein SD. Scoliosis Research Society. Multicenter spine fractures study. Spine 1992;17:528-540.

3. Smith JS, Shaffrey CI. Thoraco-lumbar injury classification for fracture - dislocations in Spine and 
DOI: 10.2478/romneu-2014-0020

spinal cord trauma - Evidence-based medicine, Thieme Medical Publishers, Inc, New York, edited by A. Vaccaro, M. F. Dvorak, 2010.

4. Reyes-Sanchez A, Rosales LM, Miramontes VP, Garin DE. Treatment of thoraco-lumbar burst fractures. Eur. Spine J. (2002) 11: 8 - 12.

5. Phadnis AS, Tan CJ, Raman AS, et al. Fracture and complete dislocation of the spine with normal motor neurology. Injury Extra. 2006; 37; 479-483.

6. VaccaroAR, Lehman RA, Hurlbert J, Anderson PA, Harris M.A New classification of thoracolumbar injuries. The importance of injury morphology, the integrity of the posterior ligamentous complex, and neurologic status. Spine Volume 30, No. 20, pp 2325-2333, 2005.

7. Lubeski D, Abdullah KG, Steinmetz MP, Masters F, Benzel EC, Mroz TE, et al. Lateral extracavitary, costotransversectomy, and transthoracic approaches to the thoracic spine: review of techniques and complications. J. Spinal Disorders Tech, Vol. 26, No. 4, June 2013.

8. Feng Wang, Ye Zhu. Treatment of complete fracturedislocation of thoraco-lumbar spine. J. Spinal Disorders Tech. 2013; 26:421-426.

9. Obeid I, Guérin P, Gille O, Gangnet N, Aurouer N, Pointillart V, Vital JM. Total vertebrectomy and spine shortening in the management of acute thoracic spine fracture dislocation: technical note and report of 3 cases. J Spinal Disord Tech. 2011 Jul;24(5):340-5.

10. Treatment of subacute thoracic spine fracturedislocation by total vertebrectomy and spine shortening: technical note. Barcelos AC, Botelho RV. J Neurosurg Spine. 2013 Feb;18(2):194-200. 